\title{
Chapter 4 \\ Some Comments on Dose Assessment for \\ Members of the Public After the Fukushima \\ Daiichi NPP Accident
}

\author{
Jiro Inaba
}

\begin{abstract}
This chapter describes the importance of dose assessment, either prospectively or retrospectively, for protection of members of the public exposed to radiation after the Fukushima NPP accident. There are three points. The first point is the implication of dose assessment. The International Commission on Radiological Protection (ICRP) has developed a system of radiation protection in which dose is the most important measure of radiological risk. In decision making regarding radiation protection, it is very important to understand the implications of dose assessment and to endeavor suitable protective measures based on the results of dose assessments. The second point is the radiological impact of the Fukushima accident. A large amount of radioactivity was released into the environment, but owing to extensive monitoring and protective measures, doses received by members of the public were fortunately not high, at the level of not causing any immediate health effects. We have much to learn from the accident, which includes (1) establishment of a better strategic system for emergency response, (2) reinforcement of environmental monitoring including in vivo counting of the human body, and (3) enhancement of better communication relevant to the accident. The third point is the effects of radiation exposure of children and infants. The protection of children in the accident aftermath has been a particular concern, and parents were extremely worried about the protection of their offspring. ICRP has provided age-dependent dose coefficients, so it was proposed that UNSCEAR should make the radiation risk of children more clear and that ICRP should revise its recommendation to include radiation protection scheme for children.
\end{abstract}

\footnotetext{
J. Inaba $(\square)$

Radiation Effects Association,

Maruishi-Daini Bldg. 5 F, 1-9-16, Kajichou, Chiyoda-ku, Tokyo 101-0044, Japan

e-mail: zb69jv@bma.biglobe.ne.jp
} 
Keywords Dose assessment $\bullet$ Dose estimation $\bullet$ Internal dose $\bullet$ External dose $\bullet$ In vivo counting $\bullet$ Environmental monitoring $\bullet$ Infant $\bullet$ Children $\bullet$ Age-dependent dose $\bullet$ Age-dependent risk

\subsection{Introduction}

On March 11 of 2011, the Great East Japan Earthquake and Tsunami affected the Tohoku District of Japan. In TEPCO's Fukushima Daiichi Nuclear Power Station, the external power supply was lost because of damage to the grid lines caused by the earthquake, and emergency diesel generators were terminated by the tsunami, which eliminated the capability to control radioactivity in reactors and the spent fuel storage pool. Consequently, a large amount of radioactive materials was released into the environment. Immediately after the release there was tremendous difficulty in managing the radioactive materials. It was extremely difficult to maintain communication, to mobilize human resources, and to procure supplies among other areas when addressing the nuclear accident that coincided with a massive natural disaster. Then, environmental radiation monitoring was initiated and various kinds of protective measures were implemented; however, radiation exposure resulted among the residents of Fukushima Prefecture and neighboring areas. Radiation exposure was initially caused by radioisotopes of iodine and short-lived radionuclides and subsequently by radiocesium from both external irradiation and internal irradiation through consumption of foods contaminated with these radionuclides.

Data and information related to dose assessment, such as in air dose rate and the concentration of radioactive materials in various environmental media after the Fukushima accident, have been reported by various organizations and disclosed by news media and the Internet. The International Symposium held on 14 December 2012 by Kyoto University provided an excellent opportunity for the integration of relevant information. Results of dose assessment for various populations have been also reported by various authors. However, no quantified and conclusive results of dose assessment have been reported by the responsible organization so far. The results are still in the process of being finalized for official presentation to the public.

In the present comments, therefore, I start by describing the implication of dose assessment in general, then I will report some results of dose assessment in the Fukushima accident. Finally, I touch on a key point of the present accident, radiation exposure of children, which seems to me extremely important for current radiation protection.

\subsection{Implication of Dose Assessment}

Ionizing radiation and radioactive materials have always been features of our environment, which means that every one in the world is exposed to a certain level of radiation from natural sources. Moreover, it is unavoidable to receive radiation from artificial sources, including medical use, in present-day life. 
Health effects of radiation are caused by the ionization that necessarily changes atoms and molecules, at least transiently, and may thus sometimes damage cells. Ionization is the process by which atoms lose or sometimes gain electrons and thus electrically charged ions. When ionizing radiation passes through matter, energy is imparted to the matter as ions are formed in biological tissues and molecular changes result. If cellular damage does occur and is not adequately repaired, it may prevent the cell from surviving or reproducing, or it may result in viable but modified cells. The damage manifests itself as radiation damage to the organs and tissues of the body. Two outcomes, deterministic effect and stochastic effect, have profoundly different implications for the organism as a whole. The probability of causing the deterministic effect will be zero at a low level of exposure, but above some level of the threshold will increase steeply to unity. Above the threshold the severity of harm will also increase with the level of exposure. The probability of a stochastic effect resulting from radiation usually increases with increments of the level of radiation exposure in a way that is roughly proportional to the level. Thus, dose (the level of exposure) is the most important measure of health risk of radiation exposure.

Radiological protection is concerned with controlling exposure to ionizing radiation so that deterministic effects are prevented and the risk of stochastic effects is limited to acceptable levels. For assessing the "level" of radiation exposures, dosimetric quantities and their units have been developed by ICRP. The fundamental dosimetric quantity in radiological protection is the absorbed dose (Gy, joules per kilogram), the energy absorbed per unit mass. The absorbed dose is defined as the average dose over a tissue or organ of the body. To combine the dosimetric quantities to risk or detriment of radiation exposure, effective dose ( $\mathrm{Sv}$ ) has been developed applying two weighting factors, a radiation weighting factor and tissue weighting factor, to the absorbed dose. The effective dose is the measure of the health risk of radiation exposure; it allows quantification of the extent of exposure of ionizing radiation from both whole-body and partial body irradiations from external radiation sources and from intakes of radionuclides. The estimated doses can be compared with recommended dose limits or reference levels for people who are occupationally exposed and for members of the public.

Radiological protection is a technology to control doses to which individuals may be exposed. ICRP has set out general principles for planning intervention in the case of a radiation emergency in Publication 60 [1]. The current ICRP recommendation extended its guidance on the application of protective measures [2], but it says that the general principle, 'do more good than harm and optimize the protection,' remains valid. Protective measures contain certain levels of nonradiological risks in general. Therefore, in an emergency exposure situation in accidental conditions and in existing exposure situations after an accident, it is extremely important to assess doses as precisely as possible based on a realistic methodology to provide reasonable protective measures such as reasonable allocation of available resources to the cases in emergency. Doses based on realistic methodology can minimize total risk from the exposure and nonradiological risk.

In the study of radiation health effects for radiation protection, it is very important to correctly assess doses each of the affected people receive; however, it is not easy to do so in cases where the exposure occurred in the past and in an emergency 
Fig. 4.1 Generic methodology for dose assessment and dose reconstruction

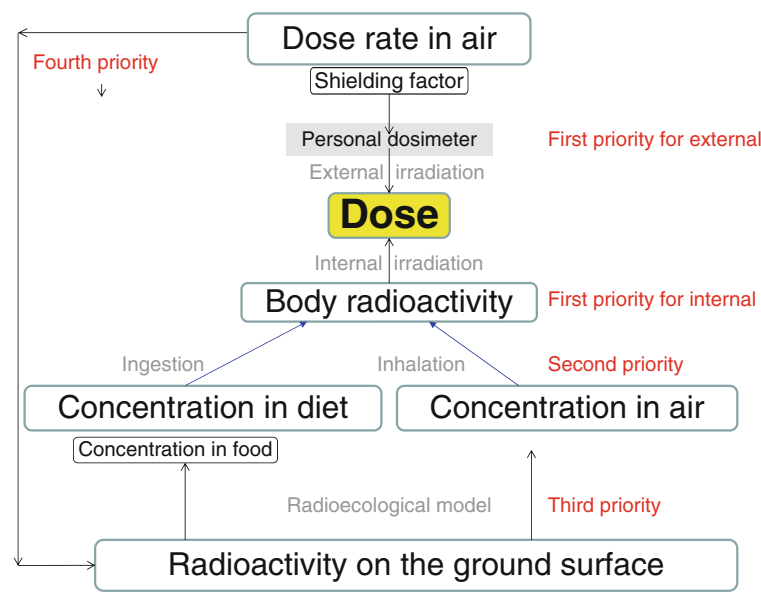

situation. Figure 4.1 shows the generic methods for dose reconstruction. In external exposure, information about the time change of the dose rate in the relevant place is important. In internal exposure, the best information is measured values for radioactivity in the human body; the second priority is radioactivity concentration in food and air. If the information is not available, we have to derive the information from the third-priority data using radioecological models.

In decision making and policy making in the fields related to radiation protection, especially in an emergency situation, it is very important for the competent organization to understand the implication of dose assessments, to appoint a proper person who knows the significance of dose assessment very clearly to a proper position in the system where decision making is done, and to devise suitable protective measures based on the results of dose assessments.

\subsection{Radiological Impact of the Fukushima Daiichi Accident}

\subsubsection{Environmental Monitoring}

Immediately after the earthquake the Japanese Government established various systems such as the Nuclear Emergency Response Headquarter to respond to the emergency. The ministry of Education, Culture, Sports, Science and Technology (MEXT) was, and currently the Nuclear Regulation Authority (NRA) is, responsible for environmental monitoring.

The accident at the Fukushima site occurred soon after the occurrence of the natural disasters of the earthquake and tsunami, which caused great difficulties in emergency response including environmental monitoring of the nuclear disaster. For instance, 23 of 24 monitoring posts in Fukushima Prefecture became unavailable, and communication and transportation of necessary resources became very 
difficult. In addition, after the loss of external power supply on March 11, TEPCO became unable to perform measurements at monitoring posts and air stack monitors, although these difficulties were resolved gradually.

In spite of the difficulties, nationwide environmental monitoring was started on March 12 by MEXT with assistance from universities and various organizations. Results of daily measurement of air dose rate, radioactivity in fallout at the fixed time, and radioactivity in tap water were reported on news media as well as Internet websites (NRA [3]). Air monitoring was done by MEXT with the help of USDOE, and a map based on air dose rate was prepared. Also, radioactivity in soil, radioactivity in seawater and ocean sediment, and radioactivity of air dust were analyzed. Measurements of foodstuffs were done by the Ministry of Health, Labor and Welfare and measurements of farmland soil and fertilizer by the Ministry of Agriculture, Forestry and Fisheries. The results of these measurements and monitorings were used for decision making of various protective measures such as evacuation and inhouse sheltering, the control of consumption and shipping of foodstuffs, as well as for dose assessment. Almost all the monitoring was done periodically, which can provide very useful information for dose assessments.

\subsubsection{Dose Assessment}

After the Fukushima accident, the public received doses through four major exposure pathways: external dose from radionuclides in the radioactive plume, external dose from radionuclides deposited on the ground, internal dose from inhalation of radionuclides in the radioactive plume, and internal dose from ingestion of radionuclides in food and water. Currently most basic information available for the dose assessment is the gamma dose rate in the air $(\mu \mathrm{Sv} / \mathrm{h})$, the density of radioactivity deposited per unit area $\left(\mathrm{Bq} / \mathrm{m}^{2}\right)$ in areas, and the concentration of radionuclides in soil samples collected $(\mathrm{Bq} / \mathrm{kg})$.

Dose assessment for individuals and groups of population is carried out using all available information.

For external dose from the ground, a dose estimation system was developed by NIRS using a time-series set of ambient dose rate maps and individual behavior with help of shielding factors of the house based on personal interview or questionnaire. External doses from a radioactive plume as well as internal dose through inhalation of the radioactive plume are calculated with help of atmospheric dispersion simulation at the time of major release of radioactivity in the affected area.

For internal dose, environmental transfer models of radioactivity from soil to human body were used. The upper soil layer, crops and vegetables, tea, milk and meat, fish and sea products, as well as tap water were important samples. Radioactivity in the total diet rather than an individual food can usually provide better information for dose assessments, although radiological survey of the total diet was not done on a wide scale by the Government after the present accident. Further, radioactivity in human body can provide the best information for dose assessment. 
Each step contains some uncertainty, so total uncertainty increases with number of steps. It means that the measured data close to the human body is very important for dose assessment with smaller uncertainty. It is very important, therefore, to emphasize that individual dosimeters for external exposure and in vivo measurements of whole body or thyroid for internal exposure can provide the best information for the dose assessments. In reality, especially in the early stages after an accident, it is not easy to make these measurements, but I think it should be emphasized.

\subsubsection{Examples of Dose Assessments in Fukushima Prefecture}

\subsubsection{External Dose}

Fukushima Prefecture has been executing a large-scale program, the "Fukushima Health Management Survey." The basic survey in the program is performed to obtain the external radiation dose which residents in Fukushima Prefecture received. A part of the results is shown in Table 4.1. The external dose is calculated by the precise map of air dose rate and the detailed time table for each resident for 4 months from March to July 2011. The total number of residents whose doses were assessed is 420,543 as of the end of March 2013; among them 411,922 are non-radiation workers. Among these non-radiation workers, $99.8 \%$ received doses less than $5 \mathrm{mSv}$, and the maximum dose was $25 \mathrm{mSv}$. The higher dose rate can be seen in highly contaminated areas [4].

As already mentioned, the external dose was calculated for just 4 months, which seems to me not long enough to look at the radiological health effect. The doses for 1 year after that accident are expedited, but it is extremely difficult to do now a similar survey on individual behavior in the past. Therefore, it would be required to calculate the 1-year dose using the ecological half-life of radiation that was shown by field monitoring. The ecological half-life of radiation is in general reported to be much shorter than the physical half-life [5].

It was reported that certain local governments distributed personal dosimeters to local residents and obtained information regarding personal dose. It is very important to compare the results of calculation and dosimeter measurement and to validate the present external dose estimation methodology. In this scene, disclosure of the related information is strongly desired.

\subsubsection{Internal Dose}

In the Fukushima Health Management Survey, whole-body counting of the residents in Fukushima Prefecture for radiocesium is also performed and the resulting internal doses were disclosed by Internet. A part of the results is shown in Table 4.2. The committed effective dose is calculated based on the measured value and 


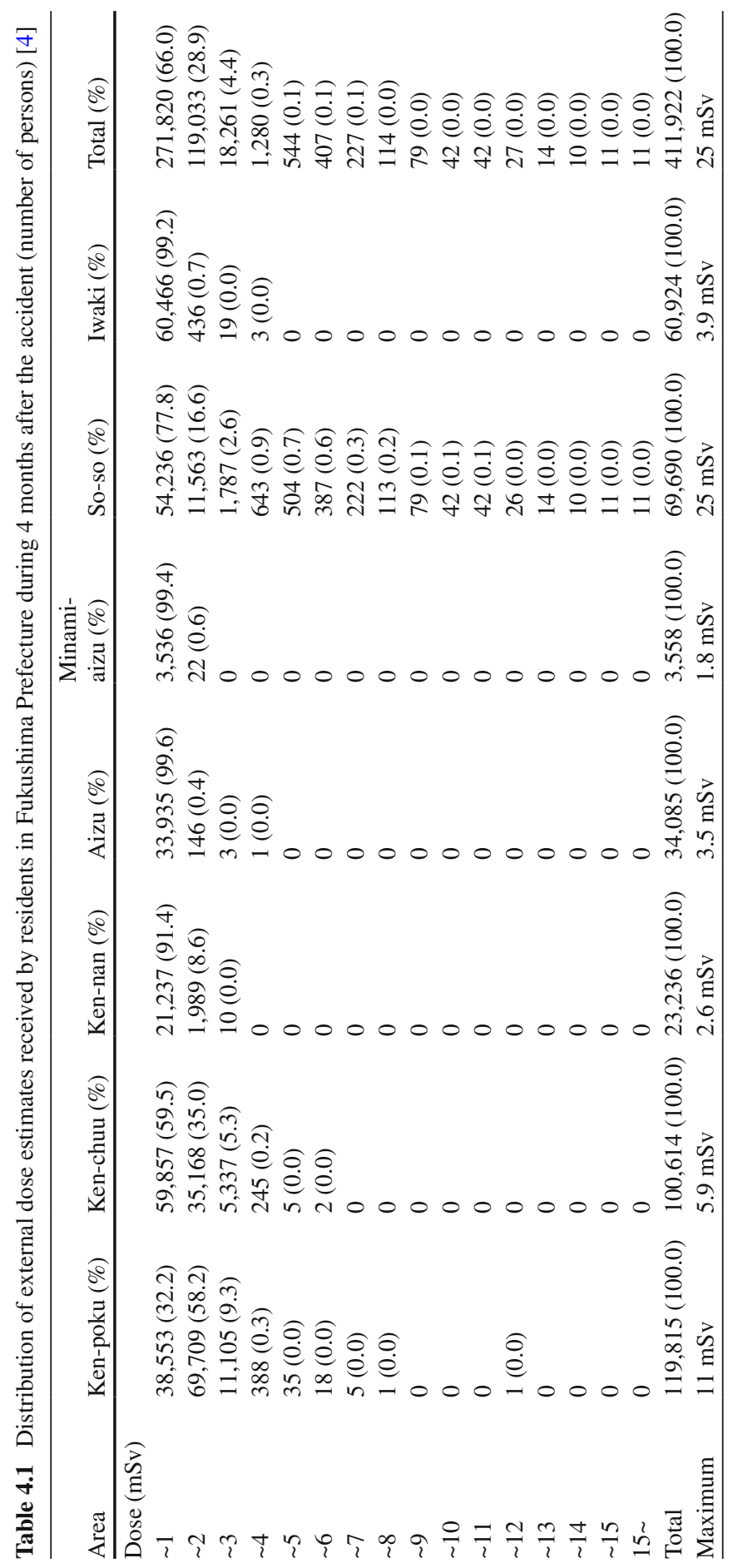


Table 4.2 Distribution of committed effective dose estimates calculated by whole-body counting of radiocesium in Fukushima Prefecture (number of persons) [7]

\begin{tabular}{lcccc}
\hline Area & Less than $1 \mathrm{mSv}$ & $1 \mathrm{mSv}$ & $2 \mathrm{mSv}$ & $3 \mathrm{mSv}$ \\
\hline Ken-poku & 34,827 & 2 & 1 & 0 \\
Ken-chuu & 31,027 & 0 & 0 & 0 \\
Ken-nan & 22,822 & 0 & 0 & 0 \\
Aizu & 5,270 & 0 & 0 & 0 \\
Minami-aizu & 692 & 0 & 0 & 0 \\
So-so & 19,673 & 12 & 9 & 2 \\
Iwaki & 17,674 & 0 & 0 & 0 \\
Total & 131,985 & 14 & 10 & 2 \\
\hline
\end{tabular}

MONDAL 3 developed by NIRS [6] on the ICRP age-dependent biokinetic model for cesium assuming scenarios of a single intake on March 12 and of chronic intake between March 12 and the counting date. From July 2011 to the end of May 2013, 132,011 people were surveyed: among them the internal dose for 131,985 was calculated to be lower than $1 \mathrm{mSv}, 14$ received $1 \mathrm{mSv}, 10$ received $2 \mathrm{mSv}$, and 2 received $3 \mathrm{mSv}$. Residents received equal to or higher than $1 \mathrm{mSv}$ live in Date City (3), Naraha Town (3), Tomioka Town (1), Kawauchi Village (1), Ookuma Town (4), Futaba Town (6), Namie Town (7), and Iitate Village (1). Date City is in Ken-poku Area and the other towns and villages are in So-so Area (5) [7].

As already mentioned the internal dose from radiocesium is a committed dose at the time of measurement, which means that the dose commitment resulting from intake of radiocesium would be a little different from the committed dose. It is interesting that all the residents whose dose was equal to or higher than $1 \mathrm{mSv}$ were detected before January 2012.

From information collected, the local government of Fukushima Prefecture concluded that the internal dose received by all residents in Fukushima Prefecture was not at a level that can affect human health.

\subsubsection{Thyroid Dose}

Apart from internal dose assessment from radiocesium, that from radioiodine is very difficult because of the short half-lives of the radioactivity as well as tissue distribution of radioiodine. Actually, in the present accident proper information was unavailable regarding internal dose assessment for the thyroid because of various reasons. In the condition of high background radiation, in vivo counting of the thyroid was very difficult. In total, 1,080 children aged 1 year to 15 years old were screened during March 24 to 30, 2011, with conventional ambient dose rate meters. Taking these data into account, it is estimated that the thyroid dose of a 1-year-old is within several tens of $\mathrm{mSv}$ and doses of other age groups are lower than that of a 1 -year-old child, although a certain extent of uncertainties in these values are unavoidable at present [8]. 
Currently, to establish methods for estimation of early internal doses to residents, various possible methods are being developed that include methods to draw a more detailed map for radioiodine using the measured data of long-lived radioiodine, ${ }^{129} \mathrm{I}$, and the radioiodine/radiocesium ratio in various environmental media, as well as atmospheric dispersion simulation with individual behavior.

\subsubsection{Some Points Found in the Dose Assessments}

The Fukushima accident is attracting attention internationally. The United Nations Scientific Committee on the Effect of Atomic Radiation (UNSCEAR) is one of the $\mathrm{UN}$ organizations responsible for scientific review of radiation exposures. In the 60th session of UNSCEAR held in May 2013, scientists discussed the Fukushima accident. Although the discussion is still in the process of finalization of presentation to the General Assembly, they issued a statement [9] concluding that "Radiation exposure following the nuclear accident at Fukushima-Daiichi did not cause any immediate health effects. It is unlikely to be able to attribute any health effects in the future among the general public and the vast majority of workers." And they reported that the exposure of the public including residents of Fukushima Prefecture is low on the whole, leading to correspondingly low risks of health effects later in life. The actions taken to protect the public significantly reduced the radiation exposures that would have otherwise been received.

In general, as UNSCEAR reported, protective actions taken so far in the disaster played important roles in the reduction of doses, but this does not mean that the actions were adequate. It is very important to learn the lessons or points to be improved from the present disaster, even though actions taken can be considered to be adequate, as much as possible.

The followings are some points raised by the author in the field of dose assessment.

(1) Establishment of better strategic systems for emergency response

In an emergency, almost all resources, including human resources, related to the radiation protection easily run short. In this situation the most important thing is to obtain information regarding the dose affected residents would receive. Of course there are cases where lifesaving should proceed, but fortunately, this is generally not the case in the present disaster. Therefore, it is very important to establish a strategic system for emergency response including protective actions based on doses that can be calculated by measurement as well as modeling. If a dose avertable by protective actions can be estimated, it would provide better information regarding the choise of protective action. In an emergency with limited resources, it is important to compromise, for instance, the number of cases of monitoring or measurement and their quality. For this purpose, the concept of the representative person developed by ICRP [10] can be used. 
(2) Reinforcement of environmental monitoring including in vivo counting of the human body

Environmental monitoring can provide essential information for radiation protection. Especially, in vivo counting of the human body can provide good information for dose assessments. However, resources for monitoring are usually limited, and moreover monitoring devices can easily be contaminated. In the present disaster, great difficulties were experienced in dose assessment for children's thyroid levels by short-lived radioiodines because in vivo counting was not performed in a precise way. This experience revealed the importance of dose assessment as well as the importance of implementation of measurement in a timely manner.

(3) Enhancement of better communication relevant to the accident

In the present accident, important information was not always provided to residents and local communities in affected areas in a timely manner. The same thing can be said in the field of dose assessments. MEXT seems for me to have a tendency to disclose information with high accuracy rather than information in a timely manner with less accuracy. In an emergency situation, it is very important to harmonize the accuracy and the timing or speed of communication. Information on dose or radioactivity, with which the public are not familiar, should be added by sufficient explanation; otherwise, rumors can spread and people's concerns can grow unreasonably as time passes.

In the end of May 2013, WHO published a report [11] on the radiological impact for the Fukushima disaster, but the risk estimation relied on rather old doses based on measurements available by mid-September 2011 [12]. It is likely that sufficient information had not been provided by the Japanese government to WHO. Actually, as already described, doses received by residents are not yet finalized as of the end of August 2013.

\subsection{Effects of Radiation Exposure of Children}

One of the characteristics of the Fukushima Daiichi Accident is the fact that people are very much concerned about the impact of radiation exposure on children.

The protection of children in the accident aftermath has been of particular concern, and parents are extremely worried about the protection of their offspring. They are suspicious that the levels of dose applied for the protection of the population as a whole do not provide sufficient safety for their offspring. The doubt may have been amplified by the natural tendency of human beings to be sensitive about children and infants. I believe that it is the time for UNSCEAR to start efforts to provide a clear summary of the current information on radiation risk of children and infants and for ICRP to start efforts for revision of their recommendation to include a radiation protection scheme for children.

ICRP has developed a radiation protection scheme for three categories of exposed individuals: workers in occupational exposure, members of the public in public exposure, and patients in medical exposure. ICRP seems to consider that the 
children are included in one of these categories and that a definite ICRP document with recommendations specifically dedicated to the protection of children and infants is not needed at present.

Because of anatomical and physiological differences, radiation exposure has a different impact on children compared with adults. UNSCEAR had started a general review of these differences before the Fukushima-Daiichi accident; its conclusions on this subject are expected to be finalized soon.

\subsubsection{Radiation Dose of Children}

There are differences in the doses received by children and adults from exposure to the same distribution of radioactive material in the environment, for example, when there are elevated levels of radionuclides on the ground. So far, ICRP has not provided age-dependent dose conversion coefficients for the members of the public [13].

Very recently ICRP organized a task group on Age-dependent Dose Conversion Coefficients for External Exposures to Environmental Source in Committee 2. The objectives of the group are to provide age-dependent dose conversion coefficients for the members of the public that are applicable to the situation where a large amount of radionuclides are released from nuclear facilities to the environment as in the present accident.

If radionuclides are ingested or inhaled, the presence of radionuclides in one organ in the human body can give higher radiation doses to another organ because the organs of children are in closer proximity to one another than those of adults. In addition, both metabolism and physiology depend on age, which also affects the biokinetics and concentrations of radionuclides in different organs and thus the dose to those organs for a given intake.

For internal exposure, the necessity for internationally accepted dose coefficients for members of the public became particularly evident after the Chernobyl reactor accident. An ICRP report entitled "Age-dependent doses to members of the public from intake of radionuclides: Part 1" was published as ICRP Publication 56 [14]. The report provided age-dependent dose coefficients $(\mathrm{Sv} / \mathrm{Bq})$ as organ equivalent dose and effective dose per unit intake of radionuclides. Then, Parts 2 to 5 were published [15-18]. Moreover, dose coefficients for embryo and fetus, as well as dose coefficients for infants through mother's breast milk, have been published as ICRP reports [19, 20]. These dose coefficients were successfully used for the internal dose assessments for the residents in the Fukushima disaster.

\subsubsection{Radiosensitivity of Children}

In contrast to radiation doses, age-dependent radiosensitivity has been treated in the current ICRP recommendations rather lightly [2]. In the 1990 recommendations of the ICRP it was reported that the total risk determined as the sum of the individual 
organ risks differs by a factor of about 3 for young (0-19 years) versus older (20-64 years) age groups [1]. For the development of radiation protection in the current ICRP recommendation, the average detriments for ages 18-64 years at exposure were used for workers and those for ages 0-85 years were used for the public.

After radiation exposure, children are reported to be more radiosensitive for about $30 \%$ of tumor types when compared with adults. These types include leukemia and thyroid, skin, and brain cancer. They have the same sensitivity as adults when it comes to $25 \%$ of tumor types such as kidney and bladder, and are less sensitive than adults when it comes to $10 \%$ of tumor types including lung cancer.

For effects that are bound to occur after high doses, UNSCEAR [9] concluded that, as seen with carcinogenesis, there are some instances in which childhood exposure poses more risk than adult exposure (e.g., for effects in the brain, cataracts, and thyroid nodules). There are other instances where the risk appears to be about the same (e.g., neuroendocrine system and effects in the kidneys), and there are a few instances where children's tissues are more resistant (lung, immune system, marrow, and ovaries).

\subsubsection{Effective Dose Adjusted by Age Weighting Factor for Radiation Protection}

Radiation exposure means the process of being exposed to radiation or radionuclides, and the significance of exposure is determined by the resulting radiation dose. Thus, dose can be considered a quantitative expression of risk of radiation exposure. In protecting individuals from the harmful effects of radiation, it is the control of radiation doses that is important, no matter what the source.

The ICRP's system of radiological protection aims primarily to protect human health. It is to manage and control exposure to radiation so that deterministic effects are prevented and risk of stochastic effects is reduced to the extent reasonably achievable.

In view of the uncertainties surrounding the values tissue weighting factors and the estimate of detriment, currently the ICRP considers it appropriate for radiological protection purposes to use age- and sex-averaged tissue weighting factors and numerical risk estimates.

However, quantitative information on the age-dependent risk coefficients of radiation exposures can be now considered to be available. Actually, WHO [11] has calculated health risk for various age groups. To control exposure so that the risk is reduced to an extent we have to develop a measure applicable to all age groups. As described previously, age-dependent dose coefficients have been already provided by ICRP. Now, it is needed for the ICRP to prepare a new report on the age-dependent radiation risks, and then to develop a new system of radiation protection for the general public to include radiation protection scheme for children based on the agedependent radiation risks. I think there are two approaches: development of new radiological protection criteria for children, and development of a new effective dose adjusted by age weighting factors. 


\subsection{Discussion}

This chapter is concerned with radiation exposure of members of the public. Other than the public, nearly 25,000 workers including TEPCO employees and contractors were also involved in the accident. Doses for workers were controlled, so no radiation-related deaths or acute effects have been observed among them. A small number of workers were highly exposed, but it is unlikely that excess cases of thyroid cancer caused by radiation exposure would be detectable. Special health examinations will be given to workers with exposures above $100 \mathrm{mSv}$.

The assessment regarding radiological effects on plants and animals was performed by UNSCEAR, and its secretary concluded that the exposures of organisms in the environment are unlikely to cause anything more than transient harm to their populations [9]. The issue is important for the environment, so detailed studies should be continued.

The experience from the 1986 Chernobyl accident has shown us that apart from any direct impact on physical health, the social and societal effects, and their associated health consequences in the affected population, are very important [21]. In this chapter, I have emphasized the importance of dose as a measure of radiation risk in radiation protection. It was reported, however, that the social and societal effects as well as their associated health consequence are not directly related to the dose. Now, we have to realize the importance of the sufficient explanation of the meaning of dose. Nevertheless, I would like to emphasize the importance of dose assessments both for practical purposes of radiation protection and for scientific goals such as epidemiological studies and radiological risk analysis.

Open Access This article is distributed under the terms of the Creative Commons Attribution Noncommercial License which permits any noncommercial use, distribution, and reproduction in any medium, provided the original author(s) and source are credited.

\section{References}

1. ICRP (1991) 1990 Recommendations of the International Commission on Radiological Protection. ICRP Publication 60. Ann ICRP 21(1-3):1-201

2. ICRP (2007) The 2007 recommendations of the International commission on radiological protection. ICRP Publication 103. Ann ICRP 37 (2-4)

3. Nuclear Regulation Authority (2013) http://radioactivity.nrs.go.jp/ja

4. Fukushima Prefecture (2013) http://www.pref.fukushima.jp/imu/kenkoukanri/25605sir you1-2.pdf

5. Pröhl G, Ehlken S, Fiedler I, Kirchner G, Klemt E, Zibold G (2006) Ecological half-lives of ${ }^{90} \mathrm{Sr}$ and ${ }^{137} \mathrm{Cs}$ in terrestrial and aquatic ecosystems. J Environ Radioact 91:41-72

6. Ishigure N, Matsumoto M, Nakano T, Enomoto H (2004) Development of software for internal dose calculation from bioassay measurements. Radiat Protect Dosim 109:235-242

7. Fukushima Prefecture (2013) http://www.pref.fukushima.jp/imu/wbc/20130628wbc_joukyou. pdf 
8. Kim E, et al (2012) Screening survey on thyroid exposure for children after the Fukushima Daiichi Nuclear Power Station Accident. In: Proceedings of the 1st NIRS symposium on reconstruction of early internal dose, NIRS, Chiba

9. UNSCEAR (2013) http://wwwunis.unvienna.org/unis/en/pressrels/2013/unisin475.html

10. ICRP (2006) Assessing dose to the representative person for the purpose of radiation protection of the public. ICRP Publication 101. Ann ICRP 346 (3)

11. World Health Organization (WHO) (2013) Health risk assessment from the nuclear accident after the Great East Japan Earthquake and tsunami based on a preliminary dose estimation. World Health Organization

12. World Health Organization (WHO) (2012) Preliminary dose estimation from the nuclear accident after the Great East Japan Earthquake and Tsunami. World Health Organization

13. ICRP (1996) Conversion coefficients for use in radiological protection against external radiation. ICRP Publication 74. Ann ICRP 26 (3/4)

14. ICRP (1989) Age-dependent doses to members of the public from intake of radionuclides: Part 1. ICRP Publication 56. Ann ICRP 20 (2)

15. ICRP (1993) Age-dependent doses to members of the public from intake of radionuclides: Part 2. ICRP Publication 67. Ann ICRP 23(3/4)

16. ICRP (1995) Age-dependent doses to members of the public from intake of radionuclides: Part 3. ICRP Publication 69. Ann ICRP 25 (1)

17. ICRP (1995) Age-dependent doses to members of the public from intake of radionuclides: Part 4. ICRP Publication 67. Ann ICRP 25 (3/4)

18. ICRP (1996) Age-dependent doses to members of the public from intake of radionuclides: Part 5. ICRP Publication 72. Ann ICRP 26 (3/4)

19. ICRP (2001) Dose to the embryo and embryo/fetus from intake of radionuclides by the mother. ICRP Publication 88. Ann ICRP 31 (1-3)

20. ICRP (2004) Doses to infants from ingestion of radionuclides in mother's milk. ICRP Publication 95. Ann ICRP 34 (3/4)

21. United Nations Scientific Committee on the Effects of Atomic Radiation (UNSCEAR) (2008) Sources and effects of ionizing radiation. UNSCEAR 2008 Report Volume 2, Annex D. United Nations Scientific Committee on the Effects of Atomic Radiation 